\title{
Attribute-Directed Top-Down Parsing
}

\author{
Karel Müller \\ Czech Technical University, Department of Computers \\ Prague, Czechoslovakia
}

\begin{abstract}
This paper deals with a method how an effective attribute-directed top-down parser and attribute evaluator can be constructed from a conditional L-attributed grammar (CLAG). The method is based on exploitation of an attribute stack in attribute evaluation and on definition of a translation scheme for CLAG.
\end{abstract}

\section{Basic Concepts and Notations}

Our definition of attribute grammars is based on [4] and [5]. An attribute grammar (AG) $G$ over a semantic domain $D$ is a context-free grammar $G_{0}=(N, \Sigma, P, S)$, the underlying context-free grammar of $G$, augmented with attributes and semantic rules. A production $p \in P$ is denoted by $p: X_{0} \rightarrow X_{1} X_{2} \ldots X_{n}$ where $X_{0} \in N$, $X_{i} \in(N \cup \Sigma)$ for all $i, 0<i \leq n,(n \geq 0)$. The semantic domain $D$ is a pair $(\Omega, \Phi)$, where $\Omega$ is a set of sets, the sets of attribute values, and the set $\{$ true,false $\}$ of boolean values, and $\Phi$ is a collection of mappings (called semantic functions of the form $f: V_{1} \times \ldots \times V_{m} \rightarrow V_{0}$, where $m \geq 0$ and $V_{i} \in \Omega, 0 \leq i \leq m$. The set of attribute symbols denoted by $A t t$ is partitioned into Inh (inherited attribute symbols) and Syn (synthesized attribute symbols). For each attribute symbol $b \in A t t$, a set $V(b) \in \Omega$ contains all possible values of the attributes corresponding to $b$.

For $X \in N, A t t(X)$ denotes the set of attribute symbols of $X$. An attribute is denoted $X . a$, where $X \in N$ and $a \in \operatorname{Att}(X)$. Inh $(X)(\operatorname{Syn}(X))$ denotes the set of inherited (synthesized) attribute symbols of $X$. We assume that the start symbol has no inherited attributes and terminals have no attributes at all.

For $X \in N$, let $\operatorname{Ord}$ define a linear ordering of the attributes of $X$ with the inherited attributes preceding the synthesized attributes. Thus, for all $X \in N, \operatorname{Ord}(X)$ is an ordering of $\operatorname{Att}(X)$ and for $b \in \operatorname{Att}(X), \operatorname{Ord}(X)(b)$ is the index of $b$ with respect to this ordering.

A production $p: X_{0} \rightarrow X_{1} X_{2} \ldots X_{n}$ has an attribute occurrence $k . b, 0 \leq k \leq n$, if $X_{k} . b$ is an attribute. An attribute occurrence $k . b$ of $p$ is called an input occurrence, if either $b \in \operatorname{Inh}$ and $k=0$, or $b \in S y n$ and $k>0$. Otherwise $k . b$ is said to be an output occurrence. For each output occurrence $k . b$ of $p$, there is exactly one semantic rule of the form $k . b:=f\left(j_{1} . a_{1}, \ldots, j_{m} . a_{m}\right)$, where every $j_{i} . a_{i}$ is an input occurrence of $p$ and $f$ is a semantic function in $\Phi$ of the type $f: V_{1} \times \ldots \times V_{m} \rightarrow V_{0}$, where $V_{0}=V(b)$ and for $1 \leq i \leq m: V_{i}=V\left(a_{i}\right)$. Notice that attribute grammars are in Bochmann normal form. An attribute grammar is $L$-attributed, if for every semantic rule $k . b:=f\left(j_{1} . a_{1}, \ldots, j_{m} . a_{m}\right)$ such that $b$ is an inherited attribute holds $j_{i}<k$ for each $i=1, \ldots, m$.

A finite set $C(p)$ of semantic conditions is associated with each production $p \in P$. A semantic condition is an expression of the form $q\left(j_{1}, a_{1}, \ldots, j_{m} . a_{m}\right)$, where every 
$j_{i} . a_{i}$ is an input occurrence of $p$ and $q$ is a boolean-valued function of the type $q: V_{1} \times \ldots \times V_{m} \rightarrow\{$ true, false $\}$, where $V_{i}=V\left(a_{i}\right), 1 \leq i \leq m$. An attribute grammar in which for all productions $p$ the set $C(p)$ of semantic conditions is empty, is called an unconditional attribute grammar. Otherwise AG is called conditional.

Let $t$ be a complete derivation tree of underlying CFG $G_{0}$ of $G, u$ its node labelled with $X$. Then for all $b \in A t t(X), u . b$ is an attribute instance attached to a node $u$. If a node $u$ has $n$ sons $u_{1}, \ldots, u_{n}$ which are labelled according to a production $p$ : $X_{0} \rightarrow X_{1} X_{2} \ldots X_{n}$, then each semantic rule $k . b:=f\left(j_{1} . a_{1}, \ldots, j_{m} . a_{m}\right)$ associated with $p$ is interpreted as an evaluation instruction $u_{k} . b:=f\left(u_{j_{1}} . a_{1}, \ldots, u_{j_{m}} . a_{m}\right)$ associated with attribute instance $u_{k} . b$, and each semantic condition $q\left(j_{1}, a_{1}, \ldots, j_{m} . a_{m}\right)$ from $C(p)$ is interpreted as a test instruction $q\left(u_{j_{1}} . a_{1}, \ldots, u_{j_{m}} . a_{m}\right)$ associated with $p$.

A derivation tree $t$ is well evaluated if all attribute instances have values according to the associated evaluation instructions, and all test instructions associated with the productions used in the tree yield true. TREES $(G)$ denotes the set of all well evaluated derivation trees of $G$. The language generated (or defined) by an AG $G$ is defined by $L(G)=\{w \mid w=\operatorname{yield}(t)$, for some $t \in T R E E S(G)\}$. Notice that $L(G) \subseteq L\left(G_{0}\right)$. For an unconditional AG $G, L(G)=L\left(G_{0}\right)$.

Let the start symbol of the underlying CFG of an AG $G$ have a distinguished synthesized attribute symbol $r$. The translation (more precisely string-to-value translation) $T(G)$ generated (or defined) by AG $G$ is the mapping from $L(G)$ to subsets of the set $V(r)$ defined by $T(G)(w)=\{x \mid x=u . r, u$ is the root of a well evaluated tree $t, r$ is its distinguished attribute and $w=y i e l d(t)\}$. This set may contain more than one element. In this case $G$ is called semantically ambiguous, otherwise $G$ is semantically unambiguous.

Throughout this paper, conditional L-attributed grammars (CLAG) are treated. It is well known that any derivation tree in CLAG can be evaluted using the one-pass evaluation strategy [2].

\section{Attribute Stack}

In order to obtain a translation defined by a L-attribute grammar for an input string, we can simulate the one-pass evaluation of a derivation tree and allocate memory for attribute instances using a stack of registers, which can hold attribute values. For an interior node $u$ labelled with $X$, and its sons $u_{1}, \ldots, u_{n}$ labelled with $X_{1}, \ldots, X_{n}$, the stack of attribute registers (attribute stack) will be used in the following way:

- Before entering a subtree with the root $u$, the top of the attribute stack consists of registers with evaluated attributes from $\operatorname{In} h(X)$ and registers with undefined values of attributes from $\operatorname{Syn}(X)$.

- After leaving this subtree, the top of the attribute stack consists of registers with attributes from $\operatorname{Syn}(X)$.

- Before evaluation of inherited attributes of $X_{i}$, the attribute stack contains registers with evaluated attributes from $\operatorname{Syn}\left(X_{i-1}\right), \ldots, \operatorname{Syn}\left(X_{1}\right), \operatorname{Inh}(X)$ and registers with undefined values of attributes from $\operatorname{Syn}(X)$. Registers for all attributes from $\operatorname{Att}\left(X_{i}\right)$ are then added to the stack, attributes from $\operatorname{Inh}\left(X_{i}\right)$ are evaluated and a subtree with the root $u_{i}$ is entered. 
- After evaluation of attributes from $\operatorname{Syn}(X)$, the attribute stack contains registers with evaluated attributes from $\operatorname{Syn}\left(X_{n}\right), \ldots, \operatorname{Syn}\left(X_{1}\right), \operatorname{Inh}(X), \operatorname{Syn}(X)$. These registers except $\operatorname{Syn}(X)$ are then removed.

Definition 1. Attribute stack.

Let $D=(\Omega, \Phi)$ be the semantic domain of a conditional L-attribute grammar $G$. Let $N$ at be the set of natural numbers, Pos the set of positive integers, $V a l=$ $V_{1} \cup \ldots \cup V_{n} \cup\{$ unde $f\}$ for all $V_{i}$ from $\Omega$ the set containing all possible attribute values including undefined value undef. An attribute stack over the domain $D$ is a data structure of the type Astack for which the following operations are defined:

empty: $\rightarrow$ Astack

push: Astack, Val $\rightarrow$ Astack

add: $\quad$ Astack, Nat $\rightarrow$ Astack

remove : Astack, Nat $\rightarrow$ Astack read: Astack, Pos $\rightarrow$ Val

write: Astack, Pos, Val $\rightarrow$ Astack

length : Astack $\rightarrow$ Nat

These operation should satisfy the following equations:

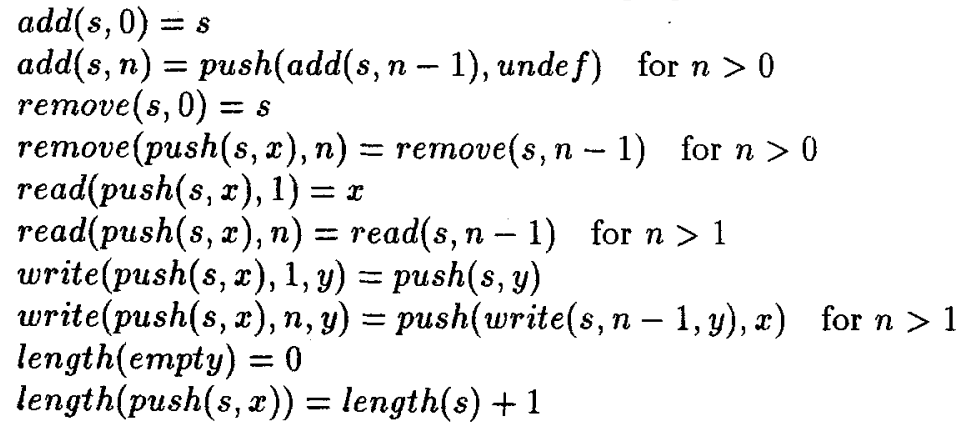

\section{Translation scheme for CLAG}

In order to formally describe an attribute evaluation using the attribute stack for a given L-attribute grammar, each semantic rule will be transformed to an operation of the type Astack $\rightarrow$ Astack and each semantic condition to an operation of the type Astack $\rightarrow\{$ true, false $\}$. Adding new registers and removing old registers will be done in the same way. These operations will be called semantic operations and semantic predicates.

For any production $p: X_{0} \rightarrow X_{1} X_{2} \ldots X_{n}$, we will define the following semantic operations and predicates:

- $A_{p, i}$ adding registers for attributes of $X_{i}, 1 \leq i \leq n$, to the attribute stack,

- $E_{p, i}$ evaluation of the inherited attributes of $X_{i}, 1 \leq i \leq n$,

- $E_{p, 0}$ evaluation of the synthesized attributes of $X_{0}$,

- $R_{p}$ removing registers with synthesized attributes of the right-hand side of $p$ and inherited attributes of the left-hand side of $p$ from the attribute stack,

- $P_{p, 0}$ a predicate which is evaluated and tested before entering a subtree with the root $X_{1}$

- $P_{p ; i}$ a predicate which is evaluated and tested after leaving a subtree with the root $X_{i}, 1 \leq i \leq n$.

Semantic operations and predicates can be constructed by the Algorithm 1. 
Algorithm 1: Construction of semantic operations and predicates.

Input: A conditional L-attributed grammar $G$.

Output: $O P$, the set of semantic operations, and $P R$, the set of semantic predicates. Method: For each production $p: X_{0} \rightarrow X_{1} X_{2} \ldots X_{n}$, let $F_{k}$ be the set of all sernantic rules $k . b:=f\left(j_{1} \cdot a_{1}, \ldots, j_{m} . a_{m}\right), 0 \leq k \leq n$, and $C_{k}$ the set of all semantic conditions $q\left(j_{1}, a_{1}, \ldots, j_{m} . a_{m}\right)$, for which $k=\max \left(j_{1}, \ldots, j_{m}\right)$. For $k=0,1, \ldots, n$ construct the semantic operations and the semantic predicates according to the following rules:

(1) For each attribute occurrence $i . a$ in $F_{k}$ define the attribute stack selector $\operatorname{sel}_{k}(i . a)$ as follows:

$$
\begin{array}{ll}
\operatorname{sel}_{k}(i . a)= & \\
\operatorname{Ord}\left(X_{k}\right)(a) & \text { if } k>0, i=k, \\
\left|\operatorname{Att}\left(X_{k}\right)\right|+\sum_{j=i+1}^{k-1}\left|\operatorname{Syn}\left(X_{j}\right)\right|+\operatorname{Ord}\left(X_{i}\right)(a)-\left|\operatorname{Inh}\left(X_{i}\right)\right| & \text { if } k>0,0<i<k, \\
\left|\operatorname{Att}\left(X_{k}\right)\right|+\sum_{j=1}^{k-1}\left|\operatorname{Syn}\left(X_{j}\right)\right|+\operatorname{Ord}\left(X_{0}\right)(a) & \text { if } k>0, i=0, \\
\sum_{j=i+1}^{n}\left|\operatorname{Syn}\left(X_{j}\right)\right|+\operatorname{Ord}\left(X_{i}\right)(a)-\left|\operatorname{Inh}\left(X_{i}\right)\right| & \text { if } k=0, i>0, \\
\sum_{j=1}^{n}\left|\operatorname{Syn}\left(X_{j}\right)\right|+\operatorname{Ord}\left(X_{i}\right)(a) & \text { if } k=0, i=0 .
\end{array}
$$$$
\text { if } k>0, i=k \text {, }
$$$$
\text { if } k>0, i=0 \text {, }
$$$$
\text { if } k=0, i>0 \text {, }
$$$$
\text { if } k=0, i=0 \text {. }
$$

(2) For each attribute occurrence $i . a$ in $C_{k}$ define the attribute stack selector $\operatorname{sel}_{k}(i, a)$ as follows:

$$
\begin{array}{ll}
\operatorname{selc}_{k}(i \cdot a)= & \text { if } k>0, i=k, \\
\operatorname{Ord}\left(X_{k}\right)(a)-\left|\operatorname{Inh}\left(X_{k}\right)(a)\right| & \text { if } k>0,0<i<k, \\
\sum_{j=i+1}^{k}\left|\operatorname{Syn}\left(X_{j}\right)\right|+\operatorname{Ord}\left(X_{i}\right)(a)-\left|\operatorname{Inh}\left(X_{i}\right)\right| & \text { if } k>0, i=0, \\
\sum_{j=1}^{k}\left|\operatorname{Syn}\left(X_{j}\right)\right|+\operatorname{Ord}\left(X_{0}\right)(a) & \text { if } \mathrm{k}=0, \mathrm{i}=0 . \\
\operatorname{Ord}\left(X_{0}\right)(a) & \text { For each semantic rule } k \cdot b:=f\left(j_{1} . a_{1}, \ldots, j_{m} . a_{m}\right) \text { define the semantic operation }
\end{array}
$$

(3) For each semantic rule $k . b:=f\left(j_{1} . a_{1}, \ldots, j_{m} . a_{m}\right)$ define the semantic operation $\operatorname{sop}_{k, b}$ as

$$
\operatorname{sop}_{k, b}(s)=\operatorname{write}\left(s, \operatorname{sel}_{k}(k . b), f\left(\operatorname{read}\left(s, \operatorname{sel}_{k}\left(j_{1} . a_{1}\right)\right), \ldots, \operatorname{read}\left(s, \operatorname{sel}_{k}\left(j_{m} . a_{m}\right)\right)\right)\right) \text {. }
$$

Construct the semantic operation $E_{p, k}$ as a composition of the operations $\operatorname{sop}_{k, b}$ : $E_{p, k}(s)=\operatorname{sop}_{k, b_{1}}\left(\operatorname{sop}_{k, b_{2}}\left(\ldots\left(\operatorname{sop}_{k, b_{m}}(s)\right) \ldots\right)\right)$.

Add $E_{p, k}$ to $O P$.

(4) For each semantic condition $q\left(j_{1} \cdot a_{1}, \ldots, j_{m} . a_{m}\right)$ from $C_{k}$ define the semantic predicate $s p r_{k, q}$ as

$$
\operatorname{spr}_{k, q}(s)=q\left(\operatorname{read}\left(s, \operatorname{selc}_{k}\left(j_{1} . a_{1}\right)\right), \ldots, \operatorname{read}\left(s, \operatorname{sel}_{k}\left(j_{m} . a_{m}\right)\right)\right) \text {. }
$$

Construct the semantic predicate $P_{p, k}$ as a conjuction of the predicates $\operatorname{spr}_{k, q}$ :

$$
P_{p, k}(s)=s p r_{k, q_{1}}(s) \text { and } \ldots \text { and } s p r_{k, q_{m}}(s) \text {. }
$$

Add $P_{p, k}$ to $P R$.

(5) If $k>0$ and $s z=\left|A t t\left(X_{k}\right)\right|$ is greater then 0 , then add to $O P$ the semantic operation $A_{p, k}$ defined as $A_{p, k}(s)=\operatorname{add}(s, s z)$.

(6) If $k=0$ and $s z=\left|\operatorname{Inh}\left(X_{0}\right)\right|+\sum_{j=1}^{n}\left|\operatorname{Syn}\left(X_{j}\right)\right|$ is greater then 0 , then add to $O P$ the semantic operation $R_{p}$ defined as $R_{p}(s)=\operatorname{remove}(s, s z)$.

Definition 2. Translation scheme for CLAG.

Let $G$ be a conditional L-attributed grammar over a semantic domain $D$ with underlying CFG $G_{0}=(N, \Sigma, P, S), O P$ the set of semantic operations, and $P R$ the set of semantic predicates constructed by the Algorithm 1. A translation scheme for $G$ is the translation grammar $Q=(N, \Sigma, \Gamma, R, S)$, where $\Gamma=O P \cup P R$ and each production $r \in R$ corresponds to one and only one production $p \in P$ in the following 
way:

$$
\begin{aligned}
& p: X_{0} \rightarrow X_{1} X_{2} \ldots X_{n} \\
& r: X_{0} \rightarrow P_{p, 0} A_{p, 1} E_{p, 1} X_{1} P_{p, 1} \ldots A_{p, n} E_{p, n} X_{n} P_{p, n} E_{p, 0} R_{p}
\end{aligned}
$$

If any of the symbols $P_{p, i}, A_{p, i}, E_{p, i}$ or $R_{p}$ does not exist then empty string is used instead of the symbol in production $r$. Any symbol from the set $\Gamma$ will be called an action symbol.

Definition 3. Attributed derivation.

Let $Q=(N, \Sigma, O P \cup P R, R, S)$ be the translation scheme of a CLAG. An attributed form (A-form) is a pair $(\alpha, s)$ where $\alpha \in \Sigma^{*}\{.\}(N \cup \Sigma \cup O P \cup P R)^{*}, s \in$ Astack. A direct attributed derivation is the relation between attributed forms denoted by $\Rightarrow$ and defined as follows:
1. $(\alpha X \beta, s) \Rightarrow(\alpha \delta \beta, s)$
2. $(\alpha . a \beta, s) \Rightarrow(\alpha a . \beta, s)$
if $X \in N, X \rightarrow \delta$ is a rule in $R$,
3. $(u . E \beta, s) \Rightarrow(u . \beta, E(s))$
if $a \in \Sigma$,
4. $(u . C \beta, s) \Rightarrow(u . \beta, s)$
if $E \in O P$,
if $C \in P R, C(s)=$ true.

The direct attributed derivation according to the first rule is called a syntax derivation, the others are called semantic derivations. Notation $f \Rightarrow^{*} g$ expresses that an A-form $g$ is derived from an A-form $f$, i.e. that there is a sequence of attributed forms $f=f_{0}, f_{2}, \ldots, f_{n}=g$, where $f_{i} \Rightarrow f_{i+1}, 0 \leq i<n$. This sequence is called an attributed derivation of the length $n$ of the A-form $g$ from the A-form $f$.

Definition 4. Let $Q=(N, \Sigma, O P \cup P R, R, S)$ be the translation scheme of a CLAG. The language generated by $Q$ is defined by

$$
L(Q)=\left\{u \mid(. S, \operatorname{add}(e m p t y,|\operatorname{Syn}(S)|)) \Rightarrow^{*}(u ., s), u \in \Sigma^{*}\right\} .
$$

The translation generated by $Q$ is the mapping from $L(Q)$ to subsets of the set $V(r)$, $r$ is the distinguished attribute of $S$, defined by

$$
T(Q)(u)=\left\{v \mid(. S, \operatorname{add}(e m p t y,|S y n(S)|)) \Rightarrow^{*}(u ., s), v=\operatorname{read}(s, \operatorname{Ord}(S)(r))\right\} .
$$

Theorem 5. Let $G$ be a conditional L-attributed grammar, $Q$ be the translation scheme for $G$. Then $L(G)=L(Q)$ and $T(G)=T(Q)$.

Proof. Can be found in [6].

\section{Nondeterministic Machine for CLAG}

The translation defined by a CLAG can be performed by a pushdown automaton with an infinite set of states. We define a pushdown automaton $M$ as a system $M=\left(K, \Sigma, \Gamma, \delta, q_{0}, Z_{0}, F\right)$ in the same way as in [1] with the only exception that the set of states $K$ may be infinite.

Theorem 6. Let $G$ be a CLAG, $r$ the distinguished synthesized attribute. There exists a pushdown automaton $M$ with potentially infinite set of states $K$, and a mapping $f$ of the type $K \rightarrow V(r)$, such that the language accepted by $M$ equals $L(G)$ and for $w \in L(G), v=T(G)(w)$ if and only if $\left(q_{0}, w, Z_{0}\right) \vdash_{M}^{*}(q, e, e)$ and $v=f(q)$.

Proof. Let $G_{0}=(N, \Sigma, P, S)$ be the underlying CFG of $G$ and $Q=(N, \Sigma, O P \cup$ $P R, R, S)$ the translation scheme for $G$. Then $M=\left(K, \Sigma, \Gamma, \delta, q_{0}, S, \emptyset\right)$ where 
$K$ is the set of all possible values of the type Astack, $\Gamma=\Sigma \cup N \cup O P \cup P R \cup\{E\}, E$ is a new symbol, $q_{0}$ is value of the operation add(empty, $\left.|\operatorname{Syn}(S)|\right)$ $\delta(q, a, a)=\{(q, e)\}$ for all $a \in \Sigma$, $\delta(q, e, X)$ contains $(e, \alpha)$ for all production $X \rightarrow \alpha \in R$, $\delta(q, e, O p)=\{(O p(q), e)\}$ for all $O p \in O P$ $\delta(q, e, \operatorname{Pr})=\{(q$, if $\operatorname{Pr}(q)$ then $e$ else $E)\}$ for all $\operatorname{Pr} \in P R$.

The mapping $f$ is defined as $f(s)=\operatorname{read}(s, \operatorname{Ord}(S)(r))$. The rest of the proof can be found in [6].

\section{Deterministic Top-down Machine for CLAG}

A deterministic top-down parser for CLAG can be driven not only by a lookahead symbol but also by conditions over attributes. Such parser is said to be attributedriven. The following definition determines a class of translation schemes for which a deterministic top-down attribute-driven parser can be constructed.

Definition 7. A translation scheme $Q=(N, \Sigma, O P \cup P R, R, S)$ of a CLAG $G$ is a ALL(1) translation scheme if for all $X \in N$ the following holds: if there are distinct productions $p_{1}: X \rightarrow \alpha_{1}$ and $p_{2}: X \rightarrow \alpha_{2}$, such that:

$F I R S T_{1}\left(\alpha_{1} . F O L L O W_{1}(X)\right) \cap F I R S T_{1}\left(\alpha_{2} . F O L L O W_{1}(X)\right) \neq \emptyset$, then $\alpha_{1}=P_{1} \beta_{1}, \alpha_{2}=P_{2} \beta_{2}, P_{1}$ and $P_{2} \in P R$, and for any value $s$ of the type Astack, for which both $P_{1}(s)$ and $P_{2}(s)$ are defined, expression $\left(P_{1}(s)\right.$ and $\left.P_{2}(s)\right)$ yields false.

Definition 8. A parse table for an ALL(1) translation scheme $Q$ is a mapping $M$ of the type $N \times(\Sigma \cup\{e\}) \rightarrow A C T$, in which $A C T$ is a set of actions containing elements $\operatorname{expand}(p), \operatorname{select}\left(p_{1}, p_{2}, \ldots, p_{n}\right)$ and error, where $p, p_{1}, \ldots, p_{n}$ are productions of $T$. - $M(X, u)=\operatorname{expand}(p)$ if $p: X \rightarrow \alpha, u \in F I R S T_{1}\left(\alpha . F O L L O W_{1}(X)\right)$ and either the first symbol of the string $\alpha$ is a predicate symbol or for any other production $X \rightarrow \beta$ holds $u \in F I R S T_{1}\left(\beta . F O L L O W_{1}(X)\right)$.

- $M(X, u)=\operatorname{select}\left(p_{1}, \ldots, p_{n}\right)$ if $p_{1}: X \rightarrow P_{1} \alpha_{1} \ldots p_{n}: X \rightarrow P_{n} \alpha_{n}$ are all Xproduction for which $P_{i}$ is in $P R$ and $u \in F I R S T_{1}\left(\alpha_{i} . F O L L O W 1(X)\right)$.

- Otherwise $M(X, u)=$ error.

Algorithm 2: ALL(1) parser for translation scheme.

Input: An ALL(1) translation scheme $Q$ for CLAG $G$ with the distinguished attribute $r$, an input string $w$.

Output: if $w \in L(G)$, then $T(G)(w)$; otherwise, an error indication.

Method: Let $M$ be the parse table for $T$. A configuration of the parser is a triple $(v, \alpha, s)$, where $v \in \Sigma^{*}$ is an unread part of the input, $\alpha \in(N \cup \Sigma \cup O P \cup P R)^{*}$ is a current content of the parsing stack and $s$ is a current value of the attribute stack. A move of the parser is the relation between configurations denoted by $\vdash$ and defined as follows:

1. $(a v, Z \alpha, s) \vdash(v, \alpha, s) \quad$ if $Z \in \Sigma, Z=a$,

2. $(a v, Z \alpha, s) \vdash(a v, \beta \alpha, s)$ if $Z \in N, M(Z, a)=\operatorname{expand}(Z \rightarrow \beta)$, 
3. $(a v, Z \alpha, s) \vdash(a v, \beta \alpha, s) \quad$ if $Z \in N, M(Z, a)=\operatorname{select}(\ldots, Z \rightarrow P \beta, \ldots)$,

$P \in P R, P(s)=$ true,

4. $(a v, Z \alpha, s) \vdash(a v, \alpha, s) \quad$ if $Z \in P R, Z(s)=t r u e$,

5. $(a v, Z \alpha, s) \vdash(a v, \alpha, Z(s))$ if $Z \in O P$.

The execution of the algorithm is as follows:

(1) Starting in the initial configuration $C_{0}=(w, S$, add (empty, $\left.|\operatorname{Syn}(S)|)\right)$, compute successive next configurations $C_{0} \vdash C_{1} \vdash \ldots \vdash \ldots$ until no further configurations can be computed.

(2) If the last computed configuration is $(e, e, s)$ then result is $\operatorname{read}(s, \operatorname{Ord}(s)(r))$. Otherwise, report an error.

Translation schemes can be transformed by transformations known for translation grammars. Therefore an ALL(1) parser can be constructed also in case the underlying CF grammar of a CLAG $G$ is not $\mathrm{LL}(1)$ but a transformation of the translation scheme for $G$ into an ALL(1) form succeeds. Moreover, special transformations for translation schemes can be developed. These transformations respect the semantics of action symbols. For more details see [6].

\section{Implementation}

The method described in the previous sections has been fully implemented in the compiler constructor ATRAG 4.0 [6]. This system was used several times as a tool supporting development and implementation of a commercial compiler. For instance, the front-end part of the Pascal compiler for processor Intel 8096 family was specified by a conditional L-attribute grammar with non LL(1) syntax. The recent practical expoitation of ATRAG is the front-end part of a translator from Hewlett-Packard Basic 5.5 into ANSI-C language.

\section{References}

[1] Aho,A.V., Ullman,J.D.: The theory of parsing, translation and compiling. Vol.1 and Vol.2, Prentice Hall, Engelwood Cliffs, N.J., 1972.

[2] op den Akker,R.: Parsing attribute grammar. Doct. Diss., Dept. Comput. Sci., University of Twente, The Netherlands, 1988.

[3] op den Akker,R., Melichar,B. and Tarhio,J.: Attribute evaluation and parsing. In: Proc. of International Summer School SAGA (ed. H.Alblas and B.Melichar), Lect. Notes Comput. Sci. 545, Springer-Verlag, Berlin, 1991, pp. 187-214.

[4] Filè,G.: The theory of attribute grammars. Doct. Diss., Twente University of Technology, Enschede, The Netherlands, 1983.

[5] Knuth,D.E.: Semantics of context-free languages. Math. System Theory 2 (1968), pp. 127-145.

[6] Müller,K.: Attribute-directed top-down parsing. Research Rep. DC-92-05, Dept. of Comp., Czech Univ. of Techn., Prague, 1992.

[7] Watt,D.A.: Rule splitting and attribute-directed parsing. In: Proc. of Workshop Aarhus (ed. N.D.Jones), Lect. Notes Comput. Sci. 94, Springer-Verlag, Berlin, 1980, pp. $363-392$. 